\title{
Pluralismo teórico y transferencia didáctica (o la literatura en la encrucijada entre el relativismo y la ortodoxia) ${ }^{1}$.
}

Analía Gerbaudo

Universidad Nacional del Litoral

\begin{abstract}
Liminar
¿Qué es "conocer literatura"? ¿Existen métodos para acceder al objeto? ¿Cómo se relacionan los campos de la literatura, la teoría y la crítica, por un lado, y la enseñanza de la literatura, por el otro? ¿Qué enseñamos cuando decimos que "enseñamos literatura"? En esta presentación pretendo defender la necesidad de una revisión de las conexiones que se establecen entre los trabajos de investigación en teoría, metodología y didáctica de la literatura, especialmente desde los espacios de formación de formadores. En primer lugar consigno un conjunto de interpelaciones enunciadas por pensadores que comparten una preocupación: desentrañar cómo las dimensiones política e ideológica atraviesan el debate teórico contemporáneo. Luego desarrollo una perspectiva teórica actual que estimo demoledora pero pertinente para pensar las relaciones que se fijan entre las construcciones de los objetos disciplinares y la fuerza de las institucionalizaciones. Finalmente concluyo con un conjunto de preguntas formuladas a partir de un trabajo de investigación que estoy realizando en el campo de la didáctica y con nuevas interpelaciones lanzadas hacia el espacio de este cruce interdisciplinar configurado por la teoría, la crítica y la transferencia educativa.
\end{abstract}

\section{Interpelaciones}

En Is there a text in this class? The autorithy of interpretative communities (1980) Stanley Fish relata una anécdota interesante: en una de sus clases escribió en la pizarra una lista de sustantivos y los presentó a sus alumnos como un poema. Tan pronto como les dijo esto, los jóvenes comenzaron a mirar al objeto con "ojos de búsqueda" de elementos poéticos, es decir, activaron sus saberes y convocaron un conjunto de expectativas acerca de lo que un poema es y de lo que con un poema se puede hacer: sus praxis habituales funcionaron como "recetas" de lo que había que buscar y de cómo había que operar, y en ese proceso, encontraron para el texto distintos sentidos.

Este relato de Fish nos obliga a preguntarnos respecto del papel de los supuestos teóricos y epistemológicos en la configuración de los saberes disciplinares: cómo 
impactan estos supuestos en la construcción de los objetos, en la fijación de las prácticas legítimas, en el establecimiento de una creencia.

En el prefacio a Las palabras y las cosas. Una arqueología de las ciencias humanas (1966), Foucault nos comenta que ese libro nace de un texto de Borges:

De la risa que sacude, al leerlo, todo lo familiar al pensamiento -al nuestro: al que tiene nuestra edad y nuestra geografía-, trastornando todas las superficies ordenadas y todos los planos que ajustan la abundancia de seres, provocando una larga vacilación e inquietud en nuestra práctica milenaria de lo Mismo y lo Otro. Este texto cita 'cierta enciclopedia china' donde está escrito que 'los animales se dividen en a) pertenecientes al Emperador, b) embalsamados, c) amaestrados, d) lechones, e) sirenas, f) fabulosos, g) perros sueltos, h) incluidos en esta clasificación, i) que se agitan como locos, j) innumerables, k) dibujados con un pincel finísmo de pelo de camello, l) etcétera, m) que acaban de romper el jarrón, n) que de lejos parecen moscas'. Es el asombro de esta taxinomia, lo que se ve de golpe, lo que, por medio del apólogo, se nos muestra como encanto exótico de otro pensamiento, es el límite del nuestro: la imposibilidad de pensar esto. Así, pues, ¿qué es imposible pensar y de qué imposibilidad se trata? (1968: p. 1).

¿Qué estudiante de letras no se maravilló cuando leyó este texto? ¿Pudo alguien vivir igual después de haberlo leído, pudo alguien pensar igual?

En Presencias reales (1989) George Steiner, a pesar de rechazar la postura deconstruccionista a la que opone como salida alternativa la filología, dice:

...Sólo podemos imaginar una teoría sistemática del significado en un sentido metafórico. El significado, en término de prueba, no es más decidible, no está más sujeto a la suspensión de la demostración experimental, que el propósito -si es que hay alguno- o 'sentido' de nuestras vidas en la ilimitada escritura del tiempo y del mundo. No podemos, en ningún modo teórico o experimental, hacer explicable mediante prueba analítica nuestro llegar a ser o nuestra muerte. Esta inexplicabilidad es la esencia de la libertad. Es la irresistible liberalidad de la imaginación y el pensamiento. La literatura, el arte y la música son las condensaciones voluntarias de esa libertad... Cuando permitimos la entrada en nuestro ser de la poesía, la música y el arte (cuando les otorgamos el 'derecho de ciudadanía' en nuestra ciudad), contemplamos la presencia desnuda -puede tener un semblante inhumano- de la propia libertad. Por lo tanto, las dudas radicales y metódicas, como la deconstrucción y la estética de las malas lecturas, están justificadas cuando niegan la posibilidad de una hermenéutica sistemática y exhaustiva, cuando niegan cualquier llegada de la interpretación a una singularidad demostrable y estable del significado; pero entre este absoluto ilusorio, esta finalidad que de hecho negaría la esencia vital de la libertad, y el juego gratuito, despótico en sí por su arbitrariedad, del sinsentido interpretativo, se encuentra el fértil y legítimo terreno de lo filológico. (1989: p. 201-202)

A pesar de que creo que la postura de Steiner es demasiado eufórica en relación a la perspectiva filológica y que vehiculiza una mala lectura de la deconstrucción, es interesante por su relativización de los criterios cientificistas a partir de los cuales se justifican algunas operaciones de interpretación.

En Benjamin o hacia una critica revolucionaria (1981), en "Universidad y Estudios culturales" (1996a) en Las ilusiones del postmodernismo (1998), en La función de la crítica (1996b) Eagleton repite sus tesis centrales: el cuestionamiento de la función social de la crítica y de la teoría literaria actuales. Estas actividades no serían sino el producto de la parálisis política y del fenómeno postmoderno: el postmodernismo subvierte los valores absolutos y algunos fundamentos metafísicos pero no repara en que las diferencias, las transgresiones, las multiplicidades a las que apela son tan nativas del capitalismo como "el pastel de manzana a los Estados Unidos" (1996: p. 19). Bajo la lógica de una aparente anarquía (lógica de placer y pluralidad, de lo efímero y lo discontinuo) no reina sino un orden político, 
ético e ideológico bastante menos flexible que la sustenta. Para Eagleton la revisión del papel de la teoría no es una cuestión solucionable en el mismo plano en que se la plantea: lo que resulte de esta crisis será una cuestión de política, no de teoría.

Si la fuerza crítica de la obra de arte moderna se perdía a causa de su distancia respecto de la vida social, la de muchos artefactos modernos lo hace por su complicidad con ella. La tendencia juguetona, populista, democrática de la cultura postmoderna constituye un justo reproche al frío elitismo de sus padres modernistas, pero a veces, y a veces al mismo tiempo, es la última e incondicional rendición a la mercancía de la que el modernismo se defendió con uñas y dientes, espesando las texturas y distorsionando las formas a fin de no ser fácilmente consumido (1996a: p. 20).

La resistencia a la teoría (1982) de Paul De Man gira en torno a la posibilidad/ imposibilidad de la teoría literaria como disciplina: las preguntas centrales del texto de De Man obligan a una revisión de los supuestos y las categorías a partir de los cuales la teoría se había consolidado como saber académico legalizado: el problema inicial con el que se enfrenta la teoría literaria es que parte de concepciones apriorísticas más que de un examen del objeto literario mismo (si es que ese objeto existe realmente). A partir de ese primer cuestionamiento replantea la relación entre "verdad" y "método" en el campo de los estudios sobre la literatura: ¿̨hay algo en la literatura que permite una discrepancia entre verdad y método? Según el decir de De Man un método que no pueda acoplarse a la verdad de su objeto pareciera que sólo puede enseñar ilusiones:

vale la pena examinar si éste es el caso [de la literatura] y por qué. Porque si es así realmente, entonces es mejor fracasar enseñando lo que no debería ser enseñado que triunfar enseñando lo que no es verdad. (1982: p. 13).

La teoría literaria debiera reflexionar respecto de los métodos de investigación sobre la literatura. Ahora bien, si los métodos no sirven para alcanzar verdades acerca de lo literario ¿en qué se basan las derivaciones de la didáctica? Y, retomando el problema anterior ¿cómo se diseñan las investigaciones para un objeto empírico antes de conocerlo?

Otra pregunta que corroe el edificio teórico existente tiene que ver con los recortes que le permitieron fijar su objeto de estudio: De Man se pregunta qué hay en Occidente detrás del proyecto que privilegió la tradición estética o la lógicogramatical en detrimento de los estudios retóricos, los únicos que permitirían una lectura teóricamente sostenible:

La resistencia a la teoría es, de hecho, una resistencia a la lectura, una resistencia que tiene quizás su forma más eficaz, en los estudios contemporáneos, en las metodologías que se llaman a sí mismas teorías de la lectura, pero que, sin embargo, evitan la función que proclaman como objeto. (1982: p. 29)

La teoría obstaculizaría los procesos de investigación, por un lado, y de enseñanza, por el otro, al exhibirle a ambas prácticas el carácter incierto de los conceptos a partir de los cuales construyen sus marcos.

Estos fragmentos no hacen sino interpelarnos desde diferentes ángulos al mostrarnos cómo en muchas ocasiones la teoría no hace sino obstaculizar las operaciones que predica: nos exhiben con descaro que nuestro seguro ordenamiento no es sino uno de los tantos posibles; relativizan los éxitos de algunas disciplinas en sus praxis cotidianas en tanto suponen aprioris que se sustentan en discursos que se aproximan más a las verdades del dogma que a las de la ciencia (cuyo nombre invocan para configurarse dentro de las formaciones discursivas aceptadas). 


\section{Stanley Fish desafía a la comunidad desde la comu- nidad: imero amague o gesto deconstruccionista?}

\subsection{Fish: primera provocación}

Si como supone Fish (1980) no habría "obras" sino únicamente la historia de sus lecturas por parte de comunidades interpretativas, como mínimo es necesario considerar la dimensión epistémico-institucional como variable en el estado de la producción y circulación de los discursos críticos.

Otro concepto sobre el que Fish pone la atención es el de "desacuerdo institucional": es llamativo que, existiendo desacuerdos institucionales, no obstante, sean discutidos y resueltos de modo regular. Los acuerdos no obedecen a la estabilidad de los objetos ni de las teorías sino, más bien, a la estabilidad de la comunidad interpretativa y al buen funcionamiento de sus reglas. La discusión, a veces, más que una actividad de demostración es una actividad de persuasión, necesariamente negociada:

The facts emerge only in the context of some point of view. It follows, then, that disagreements must occur between those who hold (or are held by) different points of view, and what is at stake in a disagreement is the right to specify what the facts can hereafter be said to be. Disagreements are not settled by the facts, but are the means by wich the facts are settled. (1980: p. 338).

Esta postura de Fish impacta en la noción clásica que se sostiene de la "teoría" en tanto enfrenta los criterios en función de los cuales se configuraron los saberes respecto a este objeto, domesticado a través de las categorías que en algún momento sirvieron de sustrato a diferentes líneas: texto, períodos, géneros, cánones, estándares, cambios, etc.. Stanley Fish habla de "comunidades interpretativas" y de "instituciones", no de "tradiciones" y de "métodos de interrogación": pensar la teoría desde esta formulación supone observarla teniendo en cuenta su carácter de actividad social y supone también que deba constantemente volverse sobre sí misma, rebelando (y revelando) cualquier intento por imponerse dogmáticamente. Este texto de Fish problematiza toda idea "ingenua" respecto de la teoría. Cualquier intento por fijar una concepción aséptica, transparente, objetiva de su metalenguaje, queda descartada: cualquier intento de naturalización aparece como un acto fascista que impone por la fuerza aquello que culturalmente construye.

\section{2. Fish: segunda provocación}

En Práctica sin teoría: retórica y cambio en la vida institucional (1989) Fish nos arroja de lleno en el contexto epistémicoinstitucional en que se desenvuelven las disciplinas.

Para este autor la teoría (en ciertas condiciones) carece de consecuencias: tal como la define, no sería sino el nombre de un conjunto de principios, reglas o procedimientos que no se relacionarían con ningún campo particular de actividad, pero que, no obstante, sería lo suficientemente general como para ser pensado como una explicación de todos los campos de actividad. Como no existen tales principios, no puede haber cosa semejante a una teoría, y algo que no existe no puede tener consecuencias.

Siguiendo el razonamiento de Fish, encontramos una diferenciación entre "teoría" y "discurso teórico": la inexistencia de la teoría (pensada en los términos expuestos más arriba) no impide que el discurso teórico funcione, es decir, que tenga consecuencias de tipo no teórico.

La distinción entre teoría y discurso teórico es la que se da entre un discurso alejado de todas las prácticas (y tal discurso no existe) y un discurso que por sí mismo es una práctica, y, por 
lo tanto, tiene consecuencias, al punto de ser influyente, respetado o difundido. Es la distinción entre lo que reclama con frecuencia la teoría - estar en una relación de autoridad o de independencia con respecto a la práctica- y la fuerza que al hacer tales afirmaciones (que son imposibles de asumir) puede haber adquirido como resultado de las condiciones existentes en una institución. Es decir, puede darse el caso de que, en una disciplina en particular, el incurrir en discurso teórico y el afirmar sus imposibles pretensiones, sean ambos estrategias eficaces e incluso obligatorias. En esa disciplina la presencia de la 'teoría' tendrá ciertamente consecuencias, pero no serán diferentes ni más predecibles que las consecuencias de cualquier forma de discurso que haya adquirido cachet y prestigio. Para decirlo con brevedad, cuando la teoría tiene consecuencias, éstas son retóricas, no teóricas; una posición teórica presentada con éxito (entiéndase, persuasivamente) nunca tendrá el efecto de modificar o revolucionar la estructura básica del pensamiento, no logrará descentrarlo con más firmeza o descentrarlo con más determinación (lo que sueña la derecha y la izquierda) pero puede tener el efecto de modificar los recursos que emplea el pensamiento para seguir su

curso contingente (aunque ordenado). (1989: p. 30)

Lo que Fish expone no es sino cómo, muchas veces, en nombre de una teoría se proclama deshacer los efectos del poder y la fuerza, mientras se producen nuevos efectos por medio de una capacidad retórica que no es sino más fuerte y poderosa que la anterior (en el caso de que la teoría logre instalarse). Para Fish, cuando esas teorías logran establecerse en una comunidad de modo que todas las cuestiones que se presentan e incluso las que se conciben son pensadas dentro de sus términos:

...la producción de discurso teórico se convierte para esa comunidad en una prolongación del sentido común. (1989: p. 43)

\section{3. ¿Datos menores? ... y algunas preguntas}

¿Será un dato menor recordar que Paul De Man tuvo que sortear varios incovenientes para poder publicar su artículo "La resistencia a la teoría"? El título del volumen ya citado aquí procede de este trabajo (el primero) que llevaba, en principio, un título diferente ("La teoría literaria: objetivos y métodos").

Este artículo fue encargado por el Comité de Investigación Literaria de la Asociación de Lenguas Modernas (MLA) para su volumen Introduction to Scholarship in Modern Languages and Literatures, donde tenía que figurar como sección de teoría literaria.

A De Man le resultó difícil escribir ese trabajo, según el encargo (pedagógico), y en su lugar escribió un ensayo en el que intentaba determinar la naturaleza de la resistencia que la teoría plantea para su propia definición. El ensayo que escribió también encontró una resistencia: se lo juzgó inapropiado para el volumen que la MLA estaba preparando, por lo que le fue encargado a Paul Henardi un ensayo completamente diferente. De Man no se quejó: simplemente cambió el título del ensayo para ofrecerlo como conferencia en el Amhest College en la primavera de 1981 y decidió poco después que ése sería el título para el volumen sobre teoría que estaba proyectando entonces ${ }^{2}$.

¿Será un dato menor revisar que en el discurso inaugural de la cátedra "Literaturas Comparadas” de la Universidad de Oxford en 1994 George Steiner no pudo dejar de expresar su alegría ante el hecho de que esta cátedra se formara? Por un lado, se expidió respecto a cómo las dimensiones éticas y políticas atraviesan esta metodología de lectura y, por el otro, cuestionó, irónicamente, las críticas que 
recibe el abordaje comparado de textos literarios. Steiner se preguntaba:

Pero, ¿es esto una materia? (...) Las breves respuestas que quiero proponer a este insistente interrogante no pueden ser sino tentativas... En el campo de las humanidades (palabra orgullosa y triste), las aspiraciones de encontrar definiciones sistemáticas terminan casi siempre en una estéril tautología. La 'teoría' tiene su significado exacto y criterios de adulterabilidad en el terreno de las ciencias. No es éste el caso de las humanidades, donde las demandas de lo 'teórico' generan como sabemos bien a nuestra costa, una jerga arrogante. En relación con la experiencia y con el juicio literario y estético, la 'teoría' no es sino una intuición objetiva o una narración descriptiva que se ha vuelto impaciente. (...)

Un compromiso persistente con las lenguas naturales, una investigación constante sobre la recepción e influencia de los textos, la conciencia de las analogías y variantes temáticas forman parte de todos los estudios literarios. En la literatura comparada, estas preocupaciones, así como sus interacciones creativas, son objeto de un énfasis especial. (...)

La generosidad que ha servido para instituir esta cátedra, la colaboración de este programa de literatura comparada y de otras ramas de estudios europeos es señal de una resolución positiva. La historia de las relaciones orgánicas entre Gran Bretaña y el continente, la historia de las relaciones -ahora decisivas para nuestro futuro- entre la Europa del Este y del Oeste, el estudio de los cimientos espirituales sobre los cuales podría levantarse una potencial comunidad europea deben tener un espacio en Oxford.

Hay en este proyecto una esperanza modesta pero auténtica. Y si existe una enfermedad crónica de la que debiera estar contagiado todo profesor, ciertamente se trataría de la esperanza. (1996: pp.131-145)

¿Y en Latinoamérica? ¿Y en el tercer mundo? ¿Qué espacio podría tener el estudio de determinados temas centrales para ser debatidos desde el contexto de nuestras universidades? ¿Qué relaciones de poder, subordinación o dominio podrían leerse a partir de los estudios comparados que Steiner avala?

¿Será un dato menor comentar que los manuales para la actual EGB, que dicen ajustarse a la nueva currícula y que parecen articular un discurso actualizado, salvo contados $\operatorname{casos}^{3}$, sostienen aún perspectivas básicamente estructuralistas? El estructuralismo fue la panacea de la pedagogía y de la despolitización: las metodologías estructuralistas no sólo confieren un conjunto de procedimientos fijos que permiten acceder a las interpretaciones legítimas sino que confían en que es posible extraer la verdad del texto, delimitada por los límites del sistema a analizar. Sistema que, por otro lado, no necesitaría la inscripción en el contexto de las relaciones sociales en las que se produce, circula o se recepciona. En nombre de la ciencia, se abortan las capacidades subversivas del texto. Se acomoda el texto igual que el cuerpo al lecho de Procusto. Mientras tanto, los docentes, tranquilos (y las instituciones también).

\section{Interpelaciones 2}

En la lección inaugural de la cátedra de Semiología Lingüística del Collège de Francia (1977) Roland Barthes rescata la importancia de suavizar las oraciones en indicativo cuando de postulaciones metodológicas, respecto a la literatura, se trata:

El método no puede referirse aquí más que al propio lenguaje en tanto lucha por desbaratar todo discurso consolidado. Por ello es justo decir que también este método es una Ficción, proposición ya adelantada por Mallarmé cuando pensaba en preparar una tesis de lingüística: 'Todo método es una ficción'... Lo que quisiera yo poder renovar en cada uno de los 
años que me sea dado enseñar aquí es la manera de presentar el curso o el seminario en pocas palabras, 'sostener' un discurso sin imponerlo: ésa será la postura metódica, la quaestio, el punto por debatir. Puesto que lo que puede resultar opresivo en una enseñanza no es finalmente el saber o la cultura que vehiculiza, sino las formas discursivas a través de las que se lo propone. Ya que esta enseñanaza tiene por objeto -como he tratado de sugerirlo-, al discurso tomado en la fatalidad de su poder, el método no puede realmente referirse más que a los medios apropiados para desbaratar, desprenderse o por lo menos aligerar dicho poder. (1978: pp. 146-147)

Yo me pregunto entonces ¿Por qué no recogemos el guante que la teoría literaria hoy nos arroja y revisamos las cerrazones desde las cuales intentamos domesticar, normalizar (en términos foucaultianos) a nuestro subversivo objeto?

No existe aproximación alguna a la literatura ajena a la teoría: existen sí, principios teóricos más o menos expuestos o mantenidos de forma más o menos consciente. ¿Por qué no asumimos este límite y esta posibilidad?

Una de las críticas que con mayor frecuencia los estudiantes de literatura plantean a la hora de estudiar teorías es precisamente la tendencia de algunas a desligarse del estudio de las obras literarias para dedicarse exclusivamente al mundo de los conceptos. Atención: puede que aquí no se estén produciendo sino algunos malentendidos. En primer lugar, cabría preguntarse si lo que problematizan los estudiantes son las operaciones de la crítica o las de la teoría. Por otro lado, es importante aceptar que la teoría, si de algo debiera ocuparse, sería de "arrojar luz" sobre el carácter construido de toda aproximación al objeto: las prácticas institucionalizadas del conocimiento tienen reglas y protocolos para determinar los objetos. Una de las funciones de los debates teóricos y metodológicos es, precisamente, la de fijar y revisar los parámetros de construcción.

Pero el debate sobre la redefinición de los parámetros o la crisis de los paradigmas teóricos existentes no implica, de ningún modo, ni un anarquismo epistemológico ni un escepticismo didáctico ni la negación de las operaciones de la crítica: es sólo un llamado de atención que intenta que se acepte la naturaleza construida del objeto y el carácter también construido de la disciplina teórica y, por lo tanto, del carácter también variable de sus verdades.

Postular que la teoría funciona de un modo autónomo no es sino otra manera de evitar la aproximación al objeto. Negarla por escéptica no es sino una forma de alejarse del debate. Afirmar que sobre los textos nada puede decirse que no los deforme no es apelar sino a los conceptos kantianos que Paul De Man (1996) caracterizara como una de las más fuertes resistencias a la teoría: la que se deriva a partir de la ideología estética que concibe a los juicios sobre el arte más allá de la esfera de la razón o del entendimiento. La ausencia de un paradigma fuerte en la teoría literaria hoy (señal de salud más que de fractura de la disciplina) se contrapone a la hegemonía editorial en el ámbito de la transferencia educativa (al menos, en nuestro país).

El consenso editorial no debiera ser el criterio a través del cual se evalúa lo pertinente y lo no pertinente a la hora de decidir qué hacer cuando se dice que se enseña literatura: los éxitos medidos a partir de la mayoría no siempre son confiables.

¿O será, tal vez, que nuestro problema es el que descaradamente plantea Barthes? Lo permanente no existe más que gracias a nuestros groseros órganos que resumen y reúnen las cosas en planos comunes, mientras que nada existe bajo esta forma. El árbol es a cada instante una cosa nueva; afirmamos la forma porque no aprehendemos la sutileza de un movimiento absoluto. (Nietzsche)

El texto sería también ese árbol cuya nominación (provisoria) debemos a la grosería de nuestros órganos. Seríamos científicos por falta de sutileza.” (1978: p. 99) 
${ }^{1}$ Este trabajo es una síntesis del escrito expuesto en la Clase Abierta "La literatura, la teoría y la crítica en el fin de siglo" (actividad de extensión realizada en el marco de las propuestas del Centro de Estudios Comparados (FaFoDoc, UNL)).

${ }^{2}$ Datos extraídos de Godzich, W.: (1998) Teoría literaria y crítica de la cultura, Valencia, Frónesis, p. 197.

${ }^{3}$ En la investigación ya mencionada (Tesis Maestría Fhuc, UNL) estamos trabajando con una muestra para mapear el estado de situación de las propuestas editoriales para la EGB (relación con las líneas de la teoría sobre la literatura, articulaciones, desarticulaciones, recortes epistemológicos, incorporaciones, exclusiones, etc). De un total de 5 editoriales seleccionadas para la muestra (Santillana, Estrada, Ediciones del Eclipse, Cántaro, Aique), sólo una presenta aspectos que abordan al texto literario más allá de las fronteras del estructuralismo (Cántaro).

\section{Bibliografía}

BARTHES, R.: (1978) El placer del texto y lección inaugural, México, S.XXI, 1993.

Bourdieu, P.: (1992) Las reglas del arte. Génesis y estructura del campo literario, Barcelona, Anagrama, 1995.

DE MAN, P: (1982) La resistencia a la teoría, Madrid, Visor, 1990.

(1996) Ideología estética, Madrid, Cátedra, 1998.

EAgleton, T.: (1981) Benjamin o hacia una critica revolucionaria, Barcelona, Paidós, 1998.

(1996a) “Universidad y Estudios culturales" en Página 30, nro. 30,

La Página, Bs. As.

(1996b) La función de la crítica, Bs. As., Paidós, 1998.

(1998) Las ilusiones del postmodernismo, Bs. As., Paidós, 1998.

FISH, S.: (1980) Is there a text in this class? The autority of interpretative communities, Harvard University Press.

(1989) Práctica sin teoría: retórica y cambio en la vida insti-tucional, Madrid, Destino, 1992.

Foucault, M.: (1966) Las palabras y las cosas. Una arqueología de las ciencias humanas, S. XXI, 1991.

Godzich, W.: (1998) Teoría literaria y critica de la cultura, Valencia, Frónesis.

Palti, E. J.: (1998) Giro lingüistico e historia intelectual, Universidad Nacional de Quilmes, Bs. As.

Steiner, G.: (1989) Presencias reales. ¿Hay algo en lo que decimos?, Bs. As., Destino, 1991.

(1996) Pasión intacta, Madrid, Siruela. 